Artículo original

\title{
Análisis de la variabilidad genética de las poblaciones de gatos domésticos (Felis catus) mediante genes del pelaje en Cartagena, Colombia
}

\author{
Yiris Montes-Díaz, Yorlenis Cardales-Barrios, Enrique Pardo-Pérez* \\ Departamento de Biología, Facultad de Ciencias Básicas, Universidad de Córdoba, Montería, Colombia
}

\begin{abstract}
Resumen
El objetivo del estudio fue determinar la diversidad y la estructura genética de las poblaciones de gatos domésticos (Felis catus) mediante los genes del pelaje en Cartagena, Colombia. Se determinó el fenotipo de 472 gatos mediante observaciones directas en recorridos por las zonas urbanas de Cartagena utilizando la nomenclatura recomendada por el Committee on Standardized Genetic Nomenclature for Cats (1968) para los marcadores fenotípicos Orange, Agouti, Tabby, Dilution, Long hair, Spotting white y Dominant white. Se calcularon los parámetros genéticos de frecuencia alélica, diversidad genética, flujo génico, equilibrio de Hardy-Weinberg y distancia genética, y se infirieron las relaciones filogenéticas entre las poblaciones de gatos. El marcador non-Agouti fue el de mayor frecuencia, mientras que los genes Tabby blotched y Dominant white presentaron los valores más bajos. Se reportó la presencia del alelo Tabby abissynian en Cartagena. La mayor parte de la diversidad genética se encontró dentro de las poblaciones (), y fue poca entre las poblaciones (); se observó un elevado flujo génico y un exceso de heterocigotos, y no hubo equilibrio de Hardy-Weinberg. Las poblaciones estaban muy relacionadas genéticamente, y se evidenció, además, una posible selección natural y artificial de los marcadores non-Agouti y Tabby abissynian.
\end{abstract}

Palabras clave: Felis catus, marcadores fenotípicos, diversidad, heterocigosidad.

Analysis of genetic variations in populations of domestic cats (Felis catus) by coat genes in Cartagena, Colombia

\begin{abstract}
The aim of this work was to determine the diversity and genetic structure of domestic cat (Felis catus) populations by fur genes in Cartagena, Colombia. We established the phenotype of 472 cats through direct observations in Cartagena's urban area using the nomenclature recommended by the Committee on Standardized Genetic Nomenclature for Cats (1968) for the phenotype markers Orange, Agouti, Tabby, Dilution, Long hair, Spotting white y Dominant white. We determined the allelic frequencies, the genetic diversity, the gene flow, the Hardy-Weinberg equilibrium, and we inferred the phylogenetic relationships. The non-Agouti marker was the most frequent, while Tabby blotched and Dominant white had the lowest values. We reported the presence of the Tabby abissynian allele in Cartagena. Most of the genetic diversity was found within populations () and few between populations (), and we observed a high gene flow and an excess of heterozygotes in the population; there was no Hardy-Weinberg equilibrium. Populations were genetically correlated, and there was evidence of a possible natural and artificial selection of the non-Agouti and Tabby abissynian markers.
\end{abstract}

Key words: Felis catus, phenotypic markers, diversity, heterozygosity.

\section{Introducción}

Los gatos domésticos (Felis catus), descendientes de los gatos silvestres (Felis libyca), se cuentan entre los más eficientes felinos depredadores de presas medianas y pequeñas. Su área natural abarcaba el Norte de África, China, India, el sur de Europa, Gran Bretaña y diversas islas del Mediterráneo, pero gracias a sus características adaptativas actualmente están ampliamente distribuidos en ambientes continentales e insulares y en una gran variedad de hábitats (Ruiz-García, et al., 2005).
Los humanos y los gatos han coexistido durante miles de años, y los primeros registros de convivencia armoniosa se remontan a alrededor del año 1500 a.C. en el antiguo Egipto (Turner \& Bateson, 1988), donde habrían explotado los recursos de una mayor concentración de roedores alrededor de granjas, depósitos de granos y basureros, y se habrían

\footnotetext{
*Correspondencia:

Enrique Pardo-Pérez, epardop@correo.unicordoba.edu.co

Recibido: 7 de junio de 2015

Aceptado: 18 de noviembre de 2015
} 
beneficiado con opciones de resguardo ya mejoradas. También habrían aprovechado la menor densidad de predadores en comparación con los terrenos circundantes y las condiciones para procrearse con más posibilidades de éxito estando cerca del hombre.

Todos los animales presentan polimorfismos, sin embargo, los gatos exhiben polimorfismos visibles relacionados con el color, el patrón y la textura de la capa, características fáciles de registrar a simple vista, lo cual hace que la recolección de datos sea un procedimiento relativamente sencillo que no requiere de técnicas y tecnologías sofisticadas; además, los gatos son una especie de elección para este tipo de estudios de población por ser animales cosmopolitas y ser una población panmíctica (Todd, 1977a).

Todd (1977a) planteó que las frecuencias genéticas de diversas poblaciones originales de gatos fueron determinadas por las migraciones humanas. Esta teoría de migración histórica se ha visto sustentada notablemente en América, con los estudios de Ruiz-García \& Álvarez (2003) y de Shostell, et al. (2005) en Norteamérica. Los estudios de genética de poblaciones en gatos son muy necesarios para revelar la historia de su evolución y para la construcción de hipótesis filogenéticas sobre las relaciones entre los alelos, sin embargo, a pesar la importancia de este tipo de estudios, la información a nivel mundial es escasa y en algunos lugares, inexistente.

Cartagena se considera la ciudad más importante del departamento de Bolívar por ser desde tiempos ancestrales puerto marítimo de profuso movimiento en Colombia y
América, lo que pudo haber incidido en la diversidad genética de las poblaciones de Felis catus. Sin embargo, no existe ningún tipo de información sobre la variabilidad de marcadores fenotípicos establecida mediante los genes del pelaje en las poblaciones de gato doméstico en Cartagena. Por tal motivo, el objetivo de este estudio fue determinar la diversidad y la estructura genética de las poblaciones de gatos domésticos (Felis catus) mediante genes del pelaje en esta ciudad.

\section{Materiales y métodos}

\section{Área de estudio}

La recolección de datos se hizo en barrios de zonas urbanas del municipio de Cartagena ubicados a $10^{\circ} 25$ > 30» de latitud norte y $15^{\circ} 32$ > 25» de longitud oeste.

\section{Recolección de datos}

Se hicieron muestreos mediante la observación directa en los barrios Alto Bosque, Chile, El Campestre, El Socorro, Fredonia, Getsemaní, Las Palmeras, Los Alpes, Los Caracoles, Los Cerezos, Nuevo Bosque, Pie de la Popa, Providencia, San Fernando, Rodeo y San José, y se hizo la clasificación fenotípica de cada uno de los 472 individuos adultos encontrados ( $\mathrm{n}=472)$; cada ruta se utilizó solo una vez para evitar repeticiones de muestreo y se verificó la presencia o ausencia de los marcadores autosómicos nonAgouti(a); Blotched tabby (Tb); Dilution (d), Long hair (l); Spotting white (S) y Dominant white (W) y del locus ligado al sexo Orange (O) (Tabla 1). Por último, se hicieron registros fotográficos de cada individuo.

Tabla 1. Descripción de los siete genes estudiados (Ruiz-García, 1994).

\begin{tabular}{lll}
\hline Locus & Alelos & Característica \\
\hline O (gen ligado al sexo) & $o$ & Silvestre; pigmentación no naranja \\
\hline A (gen autosómico) & $O$ & Mutante; toda la pigmentación es naranja; epistático para la detección del locus A \\
\hline$T$ (gen autosómico) & $A$ & Silvestre color Agouti \\
& $a$ & Mutante; color no-Agouti; un mismo color; color negro; epistático para la observación del locus T \\
\hline$D$ (gen autosómico) & $t^{+}$ & Silvestre; atigrado o Mackerel tabby; recesivo frente a $T^{u}$, pero dominante para $t^{b}$ \\
\hline$L$ (gen autosómico) & $t^{b}$ & Mutante; clásico o Blotched tabb"; recesivo \\
\hline$S$ (gen autosómico) & $D$ & Mutante; abisinio o Abyssinian tabby; dominante; este alelo es poco frecuente. \\
\hline$W$ (gen autosómico) & $L$ & Mutante; color diluido; recesivo \\
\hline & $L$ & Silvestre; pelo corto \\
\hline & $S$ & Mutante; pelo largo; recesivo \\
\hline
\end{tabular}

O: Orange; a: Non-Agouti; Tb: Blotched tabby; d: Dilution; l: Long hair; s: Spotting white; W: Dominant white 


\section{Variables de estudio}

Para el estudio de la diversidad genética de las poblaciones de gatos domésticos (Felis catus) se tuvieron en cuenta los siguientes marcadores fenotípicos (Tabla 1) propuestos por el Committee on Standardized Genetic Nomenclature for Cats (1968): O (Orange. Carácter ligado al sexo) y los loci autosómicos A (A, a; Agouti Vs. non-Agouti), T ( Ta, $t+$, tb; Abyssinian tabby Vs. Mackerel o atigrado Vs. Blotched tabby), D (D, d; full color Vs. Dilution), L (L, l; pelo corto Vs. pelo largo), $S$ ( $s^{+}, S$; no manchado de blanco Vs. manchado de blanco) y $W(w+, W$; color normal Vs. Dominant white).

\section{Análisis de población}

Las frecuencias alélicas se estimaron mediante el programa PopGene 1.31 (Yeh, et al., 1999), y se establecieron la diversidad genética de Nei correspondiente a la heterocigosidad esperada $(\mathrm{He})$ y la heterocigosidad de la población total $\left(\mathrm{H}_{\mathrm{T}}\right)$, el coeficiente de diferenciación genética $\left(\mathrm{G}_{\mathrm{ST}}\right)$, el flujo génico $(\mathrm{Nm})$, el equilibrio de Hardy-Weinberg y la distancia genética entre poblaciones propuestas por Nei (1972).

Para determinar las relaciones genéticas entre las poblaciones analizadas se construyeron árboles filogenéticos mediante el algoritmo matemático de los grupos pareados sin ponderar usando la media aritmética (Unweighted Pair Group Method with Arithemetic Mean, UPGMA), con el programa MEGA 5.2 (Tamura, et al., (2011), y a partir de la matriz de distancias genéticas.

\section{Resultados}

\section{Frecuencias alélicas}

Al calcular las frecuencias alélicas para la población total se encontró que la más alta la obtuvo el alelo non-Agouti, especialmente en las subpoblaciones de Getsemaní $(0,874)$, Los Cerezos $(0,837)$ y El Campestre $(0,816)$, en tanto que los loci Tabby abissynian y Dominant white presentaron frecuencias muy bajas, siendo el locus Tabby abissynian el de menor promedio de frecuencia alélica $(0,010)$, en comparación con los loci restantes en las 16 subpoblaciones analizadas (Tabla 2).

\section{Diversidad genética}

Las subpoblaciones que presentaron los valores más altos de heterocigocidad fueron las de Alto Bosque $(\mathrm{He}=0,500)$, Chile $(\mathrm{He}=0,500)$, Los Cerezos $(\mathrm{He}=0,500)$ y El Rodeo $(\mathrm{He}=0,500)$ para el marcador Spotting white, mientras que el marcador Dominant white $(0,025)$ presentó los valores más bajos de heterocigocidad (Tabla 3). El test de HardyWeinberg (Tabla 4) a nivel global reveló ausencia de equilibrio para las poblaciones estudiadas.

\section{Diferenciación genética y flujo génico}

La diversidad genética total $\left(\mathrm{H}_{\mathrm{T}}\right)$ fue moderada, con un promedio de 0,356 , y un intervalo de 0,026 en el locus White, y de 0,498 en el locus Spotting white. La mayor parte de la diversidad genética se registró dentro de las poblaciones $\left(\mathrm{H}_{\mathrm{s}}=0,326\right)$, y se encontró poca entre las poblaciones

Tabla 2. Frecuencias alélicas de las subpoblaciones de Felis catus analizadas en Cartagena

\begin{tabular}{|c|c|c|c|c|c|c|c|c|c|}
\hline & $\mathbf{N}$ & $O$ & $a$ & $T^{a}$ & $t^{b}$ & $d$ & $l$ & $S$ & $W$ \\
\hline Alto Bosque & 21 & 0,421 & 0.795 & 0.034 & 0.258 & 0.324 & 0.309 & 0.500 & 0.049 \\
\hline Chile & 36 & 0,400 & 0.793 & 0.000 & 0.000 & 0.293 & 0.236 & 0.514 & 0.014 \\
\hline El Campestre & 36 & 0,417 & 0.816 & 0.000 & 0.218 & 0.333 & 0.289 & 0.542 & 0.000 \\
\hline El Socorro & 20 & 0,300 & 0.806 & 0.000 & 0.000 & 0.316 & 0.000 & 0.700 & 0.000 \\
\hline Fredonia & 23 & 0,391 & 0,722 & 0,000 & 0,365 & 0,466 & 0,209 & 0,543 & 0,000 \\
\hline Getsemaní & 18 & 0,588 & 0,874 & 0,000 & 0,542 & 0,243 & 0,236 & 0,529 & 0,028 \\
\hline Las Palmeras & 5 & 0,324 & 0,700 & 0,000 & 0,297 & 0,198 & 0,243 & 0,539 & 0,000 \\
\hline Los Alpes & 32 & 0,550 & 0,775 & 0,000 & 0,324 & 0,258 & 0,177 & 0,483 & 0,032 \\
\hline Los Caracoles & 39 & 0,167 & 0,577 & 0,000 & 0,000 & 0,358 & 0,226 & 0,641 & 0,000 \\
\hline Los Cerezos & 20 & 0,325 & 0,837 & 0,000 & 0,000 & 0,000 & 0,224 & 0,500 & 0,000 \\
\hline Nuevo Bosque & 30 & 0,517 & 0,788 & 0,000 & 0,000 & 0,371 & 0,322 & 0,552 & 0,017 \\
\hline Pie de la Popa & 10 & 0,667 & 0,667 & 0,106 & 0,000 & 0,471 & 0,632 & 0,556 & 0,051 \\
\hline Providencia & 22 & 0,364 & 0,674 & 0,017 & 0,775 & 0,400 & 0,213 & 0,480 & 0,000 \\
\hline San Fernando & 44 & 0,511 & 0,739 & 0,000 & 0,192 & 0,302 & 0,213 & 0,523 & 0,000 \\
\hline Rodeo & 30 & 0,400 & 0,707 & 0,000 & 0,000 & 0,258 & 0,183 & 0,500 & 0,000 \\
\hline San José & 40 & 0,359 & 0,698 & 0,000 & 0,000 & 0,160 & 0,158 & 0,423 & 0,017 \\
\hline Prom/Total & 472 & 0,419 & 0,748 & 0,010 & 0,186 & 0,297 & 0,242 & 0,533 & 0,013 \\
\hline
\end{tabular}

$N$ (tamaño de la muestra), O (Orange), a (no Agouti), (Tabby abissynian), (Tabby blotched), d (No diluido), l (Long hair), S (Spotting White) y W (Dominant White). 
Tabla 3. Heterocigocidad esperada de la población de Felis catus de Cartagena

\begin{tabular}{|c|c|c|c|c|c|c|c|}
\hline & Orange & Agouti & Tabby & Dilution & Long hair & Spotting white & Dominant white \\
\hline Alto Bosque & 0,488 & 0,326 & 0,431 & 0,438 & 0,427 & 0,500 & 0,093 \\
\hline Chile & 0,480 & 0,329 & 0,000 & 0,414 & 0,360 & 0,500 & 0,028 \\
\hline El Campestre & 0,486 & 0,300 & 0,341 & 0,444 & 0,411 & 0,497 & 0,000 \\
\hline El Socorro & 0,420 & 0,312 & 0,000 & 0,432 & 0,000 & 0,420 & 0,000 \\
\hline Fredonia & 0,476 & 0,401 & 0,464 & 0,498 & 0,330 & 0,496 & 0,000 \\
\hline Getsemaní & 0,484 & 0,220 & 0,496 & 0,367 & 0,360 & 0,498 & 0,055 \\
\hline Las Palmeras & 0,438 & 0,420 & 0,418 & 0,318 & 0,367 & 0,497 & 0,000 \\
\hline Los Alpes & 0,495 & 0,349 & 0,438 & 0,383 & 0,291 & 0,499 & 0,061 \\
\hline Los Caracoles & 0,278 & 0,488 & 0,000 & 0,460 & 0,350 & 0,460 & 0,000 \\
\hline Los Cerezos & 0,439 & 0,273 & 0,000 & 0,000 & 0,347 & 0,500 & 0,000 \\
\hline N/Bosque & 0,499 & 0,334 & 0,000 & 0,467 & 0,436 & 0495 & 0,033 \\
\hline Pie de la Popa & 0,444 & 0,444 & 0,189 & 0,498 & 0,465 & 0,494 & 0,097 \\
\hline Providencia & 0,463 & 0,439 & 0,356 & 0,480 & 0,335 & 0,499 & 0,000 \\
\hline San Fernando & 0,500 & 0,386 & 0,311 & 0,421 & 0,335 & 0,499 & 0,000 \\
\hline Rodeo & 0,480 & 0,414 & 0,000 & 0,383 & 0,298 & 0,500 & 0,000 \\
\hline San José & 0,460 & 0,422 & 0,000 & 0,269 & 0,266 & 0,488 & 0,033 \\
\hline Media & 0,458 & 0,366 & 0,215 & 0,392 & 0,336 & 0,490 & 0,025 \\
\hline
\end{tabular}

Tabla 4. Equilibrio Hardy- Weinberg mediante la prueba de bondad de ajuste de ji al cuadrado

\begin{tabular}{ccccc}
\hline Población & Locus & $\mathbf{x}^{\mathbf{2}}$ & Grados de libertad & $\mathbf{p}$ \\
\hline \multirow{2}{*}{ Cartagena } & $O$ & 16,891 & 1 & 0,0002149 \\
& $S$ & 23,692 & 1 & $7,1686 \mathrm{E}-06$ \\
\hline
\end{tabular}

O: Orange; S: Spotting white

$\left(D_{\mathrm{ST}}=0,030\right)$, lo cual indica que las 16 poblaciones compartían una gran proporción de la diversidad total. El coeficiente de diferenciación genética $\left(\mathrm{G}_{\mathrm{ST}}=0,083\right)$ indicó que $8,3 \%$ de la diversidad genética se encontraba entre las poblaciones y $91,7 \%$, dentro de las poblaciones. El flujo de genes $\mathrm{Nm}$ resultó alto $(\mathrm{Nm}=5,525)$ (Tabla 5).

\section{Distancia genética}

La distancia genética entre las poblaciones fue baja; las poblaciones de Alto Bosque y El Campestre, las de Chile y Rodeo y las de San José y El Rodeo, fueron las más cercanas, mientras que las poblaciones de Providencia y Pie de la Popa resultaron ser las de mayor diferencia génica (Tabla 6).

El dendrograma de UPGMA elaborado a partir de los valores de distancia genética de Nei (1972) para las 16 poblaciones reveló dos grupos diferenciados, uno que reunía las poblaciones de Getsemaní y Providencia, y el otro agrupaba las restantes poblaciones (Figura 1).

A nivel nacional, el dendrograma de UPGMA elaborado a partir de los valores de distancia genética de Nei (1972) ha revelado la presencia de dos grupos: uno conformado por poblaciones del interior del país como Ibagué,
Tabla 5. Distribución de la diversidad genética de la población de Felis catus de Cartagena

\begin{tabular}{lccccr}
\hline & $\mathbf{H}_{\mathbf{T}}$ & $\mathbf{H}_{\mathbf{S}}$ & $\mathbf{G}_{\mathbf{S T}}$ & $\mathbf{D}_{\mathbf{S T}}$ & $\mathbf{N m}$ \\
\hline Orange & 0,487 & 0,458 & 0,059 & 0,029 & 7.993 \\
Aguti & 0,377 & 0,366 & 0,029 & 0,011 & 16.868 \\
Tabby & 0,318 & 0,215 & 0,324 & 0,103 & 1.045 \\
Dilution & 0,418 & 0,392 & 0,061 & 0,026 & 7.683 \\
Long hair & 0,367 & 0,336 & 0,083 & 0,030 & 5.554 \\
Spotting white & 0,498 & 0,490 & 0,016 & 0,008 & 31.756 \\
White & 0,026 & 0,025 & 0,024 & 0,001 & 20.485 \\
Media & 0,356 & 0,326 & 0,083 & 0,030 & 5.525 \\
\hline
\end{tabular}

$\mathrm{H}_{\mathrm{T}}=$ diversidad genética total; $\mathrm{H}_{\mathrm{S}}=$ diversidad dentro de las poblaciones; $\mathrm{D}_{\mathrm{ST}}=$ diversidad entre poblaciones; $\mathrm{G}_{\mathrm{ST}}=$ coeficiente de diversidad genética; $\mathrm{Nm}=$ número de migrantes

Bucaramanga, Bogotá, Cali y Pasto, a las que se han unido Montería y Lorica, y un segundo grupo formado por poblaciones del Caribe colombiano como Cartagena, San Antero, Coveñas y Tolú (Figura 2).

\section{Discusión}

La frecuencia elevada del gen non-Agouti podría estar relacionada con factores ambientales como las elevadas temperaturas, que estarían favoreciendo no solo la presencia sino el aumento de los individuos que lo portan (RuizGarcía, et al., 2005; Kaelin, et al., 2012), así como con la posible existencia de sucesos selectivos asociados a una progresiva melanización del pelaje facilitados por el hecho 
Tabla 6. Matriz de distancia genética (Nei, 1972) entre subpoblaciones de Cartagena

\begin{tabular}{|c|c|c|c|c|c|c|c|c|c|c|c|c|c|c|c|c|}
\hline & 1 & 2 & 3 & 4 & 5 & 6 & 7 & 8 & 9 & 10 & 11 & 12 & 13 & 14 & 15 & 16 \\
\hline \multicolumn{17}{|l|}{1} \\
\hline 2 & 0,017 & ------ & & & & & & & & & & & & & & \\
\hline 3 & 0,002 & 0,011 & ------ & & & & & & & & & & & & & \\
\hline 4 & 0,042 & 0,018 & 0,031 & ------ & & & & & & & & & & & & \\
\hline 5 & 0,012 & 0,036 & 0,012 & 0,044 & ------ & & & & & & & & & & & \\
\hline 6 & 0,027 & 0,074 & 0,034 & 0,096 & 0,033 & ------ & & & & & & & & & & \\
\hline 7 & 0,010 & 0,023 & 0,011 & 0,037 & 0,019 & 0,037 & ------ & & & & & & & & & \\
\hline 8 & 0,010 & 0,028 & 0,012 & 0,048 & 0,018 & 0,014 & 0,016 & ------ & & & & & & & & \\
\hline 9 & 0,046 & 0,025 & 0,038 & 0,024 & 0,048 & 0,131 & 0,034 & 0,071 & ------ & & & & & & & \\
\hline 10 & 0,036 & 0,017 & 0,031 & 0,036 & 0,072 & 0,085 & 0,026 & 0,043 & 0,046 & ------ & & & & & & \\
\hline 11 & 0,019 & 0,006 & 0,013 & 0,033 & 0,039 & 0,074 & 0,037 & 0,032 & 0,039 & 0,036 & ------ & & & & & \\
\hline 12 & 0,062 & 0,063 & 0,060 & 0,128 & 0,088 & 0,121 & 0,098 & 0,086 & 0,101 & 0,113 & 0,034 & ------ & & & & \\
\hline 13 & 0,070 & 0,147 & 0,083 & 0,157 & 0,043 & 0,040 & 0,065 & 0,064 & 0,157 & 0,172 & 0,157 & 0,206 & ------ & & & \\
\hline 14 & 0,007 & 0,011 & 0,005 & 0,030 & 0,016 & 0,034 & 0,013 & 0,006 & 0,042 & 0,031 & 0,012 & 0,061 & 0,090 & ------ & & \\
\hline 15 & 0,021 & 0,002 & 0,016 & 0,018 & 0,038 & 0,079 & 0,021 & 0,028 & 0,021 & 0,016 & 0,011 & 0,074 & 0,145 & 0,011 & ------ & \\
\hline 16 & 0,028 & 0,008 & 0,025 & 0,027 & 0,051 & 0,086 & 0,022 & 0,033 & 0,029 & 0,010 & 0,024 & 0,097 & 0,152 & 0,019 & 0,004 & ----. \\
\hline
\end{tabular}

1: Alto Bosque, 2: Chile, 3: El Campestre, 4: El Socorro, 5: Fredonia, 6: Getsemaní, 7: Las Palmeras, 8: Los Alpes, 9: Los Caracoles, 10: Los Cerezos, 11: Nuevo Bosque, 12: Pie de la Popa, 13: Providencia, 14: San Fernando, 15: Rodeo, 16 San José

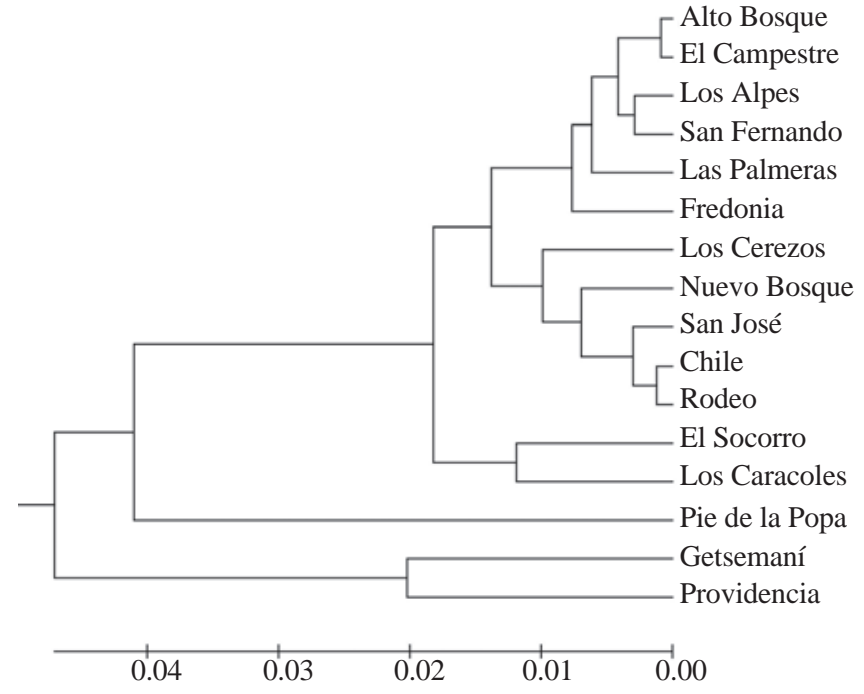

Figura 1. Dendrograma construido con el método de UPGMA con base en la distancia genética de Nei (1972) de poblaciones de Felis catus de Cartagena

de que las coloraciones melánicas se ven favorecidas en el medio urbano (Ruiz-García \& Álvarez, 2003). Algunos estudios en ratas (Keeler, 1942, 1947), en visones (Keeler \& Moore, 1961) y en zorros (Belyaev \& Trut, 1975; Keeler, 1975; Keeler, et al., 1968, 1970) han revelado una correlación entre los genes de la coloración y ciertos rasgos del comportamiento, por ejemplo, los individuos más melánicos son menos temerosos, menos agresivos y más resistentes al estrés que producen las zonas urbanas.
Por otro lado, el marcador Tabby abissynian, que no se había reportado antes para la región Caribe, presentó la menor frecuencia. La presencia de este alelo revela la introducción de este carácter africano, como lo reportaron Ruiz-García, et al. (2005) en México, y puede deberse a algún tipo de selección artificial, es decir, haber sido escogido por razones de índole físico o estético por los humanos. En este sentido, según Todd (1977a), algunos aspectos que inciden en su selección por parte de los humanos incluyen la selección por novedad, es decir, la atracción humana por lo extraño o raro, o la migración selectiva, es decir, que los exploradores o cierta población de humanos tuviera predilección por cierto tipo de carácter en los gatos.

El otro marcador de menor frecuencia fue el Dominant white, sobre el cual Ruiz-García \& Álvarez (1999) han planteado que la escasez del locus White puede usarse como medidor de la diversidad genética y para detectar la magnitud de intervención humana en las poblaciones de gatos. Además, el hecho de que la frecuencia del alelo W sea tan baja puede atribuirse a efectos pleiotrópicos sobre la audición (Geigy, et al., 2007), lo cual podría causar serias dificultades a los gatos, e incluso, una muerte temprana.

La presencia de todos los marcadores estudiados en la población de gatos domésticos de Cartagena demuestra la gran variedad de genes presente en la zona, situación que se puede atribuir a la ubicación geográfica estratégica de dicha población, ya que desde hace mucho tiempo es paso obligado de migrantes, lo que ha facilitado un considerable flujo génico. 
La diversidad genética total $\left(\mathrm{H}_{\mathrm{T}}\right)$ encontrada en Cartagena fue moderada. La mayor parte se encontró dentro de las poblaciones $\left(\mathrm{H}_{\mathrm{s}}\right)$, y entre las poblaciones $\left(\mathrm{D}_{\mathrm{ST}}\right)$ fue poca, lo cual indica que las subpoblaciones locales comparten una gran proporción de la diversidad total. El flujo de genes $\mathrm{Nm}$ resultó alto, y en ese sentido debe recordarse que los valores de $\mathrm{Nm}$ cercanos o mayores a 5 se consideran suficientes para mantener una relativa homogeneidad del acervo de genes (Slatkin, 1987). Estos resultados indicaron que, aparentemente, no hay diferenciación entre las subpoblaciones muestreadas. La falta de diferenciación genética entre poblaciones se considera generalmente como el resultado de un suficiente flujo de genes debido a las migraciones, y ocurre en todas las poblaciones para contrarrestar los efectos de la selección o la deriva genética. Según Lowe, et al., (2004), cuando Nm es mayor a 1 se espera que las poblaciones conserven conectividad genética, ya que el flujo genético sobrepasa los efectos de la deriva e impide la diferenciación local.

Un grado elevado de flujo génico (Tabla 5) permite inferir que las poblaciones se encuentran muy relacionadas genéticamente y se comportan como una metapoblación, situación a la cual se atribuye la aproximación de todas las poblaciones desde el punto de vista estructural (RuizGarcía, et al., 2005).

La desviación del equilibrio de Hardy-Weinberg para los alelos O (Orange) y S (Spotting white) puede responder a diversas causas, y dado que los resultados evidenciaron un exceso de heterocigotos y un déficit de homocigotos, esta podría atribuirse básicamente a la falta de endogamia por la existencia de un flujo génico diferencial para uno y otro sexo, y el cruce rápido de hembras y machos migrantes con los individuos residentes, lo que produciría un exceso de heterocigotos (Chesser, et al., 1993, Ruiz-García, 2010a), porque tanto machos como hembras tendrían genotipos pertenecientes a acervos genéticos altamente diferenciados; también podría responder al hecho de que estos marcadores se encuentren ligados a algún otro gen que esté afectado por heterosis y que arrastre a los marcadores a mostrar un exceso de heterocigotos, o a el efecto Wahlund, la mutación o la deriva génica.

En el dendrograma de los UPGMA elaborado a partir de los valores de distancia genética de Nei (1972) se ve que la de Cartagena se comporta genéticamente como una sola población (Figura 1), y al compararla con otras ciudades colombianas analizadas (Figura 2), se encontró similitud entre ellas, sobre todo entre Cartagena, San Antero, Coveñas y Tolú, lo que evidencia el vertiginoso desplazamiento de los conquistadores españoles por el río Magdalena, y el establecimiento de muchas ciudades y villas en poco tiempo (Ruiz-García, et al., 2005; Peña-Cruz, et al., 2015). Además, este gran parecido genético entre las poblaciones puede corresponder a un evento fundador común a partir de las poblaciones españolas (Ruiz-García, et al., 2005), pues la especie no existía en América hasta la inmigración de los europeos y su entrada debió estar asociada a las rutas colonizadoras de las naciones europeas en el Nuevo Mundo (Ruiz-García \& Álvarez, 1999). Sumado a esto cabe resaltar la similitud de algunas condiciones ambientales como la temperatura y la humedad relativa en estas ciudades.

\section{Conclusión}

El marcador con la mayor frecuencia fue el non-Agouti, lo cual podría estar relacionado con factores como las elevadas temperaturas, y el marcador que presentó la menor frecuencia fue el Tabby abissynian, el cual se reporta por primera vez para la región Caribe. La presencia de todos los genes estudiados en la población de gatos de Cartagena demuestra la gran variedad de genes presentes en la zona. La diversidad genética total encontrada en Cartagena fue moderada, y la mayor parte se registró dentro de las poblaciones, y fue poca entre las poblaciones, lo cual revela que las poblaciones locales comparten una gran proporción de la diversidad total. No se encontró, además, el equilibrio de Hardy-Weinberg para los alelos O (Orange) y S (Spotting white). El dendrograma (Figura 1) demostró que genéticamente la población de gatos de Cartagena se comportó como una sola. Las poblaciones de Cartagena, San Antero, Coveñas y Tolú (Figura 2) están muy relacionadas genéticamente, situación que puede deberse al vertiginoso desplazamiento de los conquistadores españoles por el río Magdalena y al establecimiento de muchas ciudades y villas en poco tiempo, así como a su cercanía geográfica; además, se evidenció una posible selección natural y artificial de los marcadores non-Agouti y Tabby abissynian.

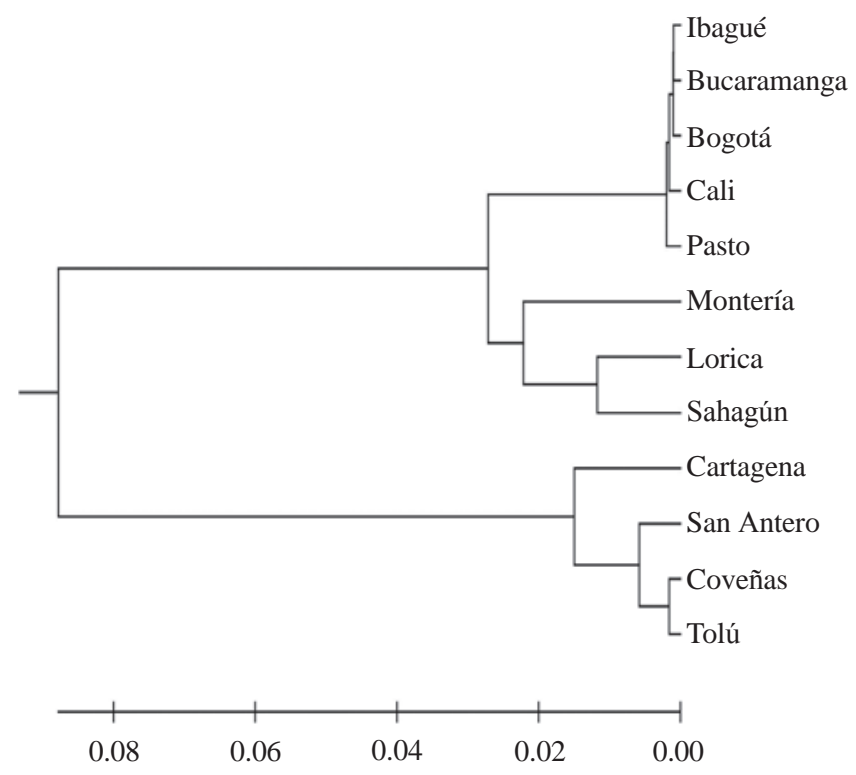

Figura 2. Dendrograma construido con el método de UPGMA con base en la distancia genética de Nei (1972) de poblaciones de Felis catus de Colombia 


\section{Conflicto de intereses}

Los autores declaran que no existen conflictos de intereses relacionados con el contenido del presente artículo.

\section{Bibliografía}

Belyaev, D. K. \& Trut, L. N. (1975). Some genetic and endocrine effects of selection for domestication in silver foxes. In: W. M. Fox (editors). The wild canids: Their systematics, behavioral ecology, and evolution. Van Nostrand Reinhold, New York. p. 416-426.

Chesser, R.K., Sugg, D.W., Rhodes, O.E., Novak, J.N., Smith, M.H. (1993). Evolution of mammalian social structure. Acta Theriol. 38: 163-174.

Commitee on Standardized Genetic Nomenclature for Cats. (1968). Standardized genetic nomenclature for the domestic cat. J. Hered. 59 (1): 39-40.

Geigy, C.A., Heid, S., Steffen, F., Danielson, K., Jaggy, A., Gaillard, C. (2007). Does a pleiotropic gene explain deafness and blue irises in white cats? Vet J. 173: 548-53.

Kaelin, C.B., Xu, X., Hong, L.Z., David, V.A., McGowan, K.A. (2012). Specifying and sustaining pigmentation patterns in domestic and wild cats. Science. 337: 1536-1541.

Keeler, C. (1942). The association of the black (non-agouti) gene with behaviour in Norway rat. J. Hered. 33: 371-384.

Keeler, C. (1947). Modification of brain and endocrine glands, as an explanation of altered behaviour trends, in coat-character mutant strains of the Norway rat. J. Tenessee Acad. Sci. 12: 202-209.

Keeler, C. \& Moore, L. (1961). Psychosomatic synthesis of behavior trends in the taming of mink. Bull. Geor. Acad. Sci. 19: 66-74.

Keeler, C., Ridgway, S., Lipscomb, L., Fromm E. (1968). The genetics of adrenal size and tameness in colour phase foxes. J. Hered. 59: 82-94.

Keeler, C.E., Mellinger T., Fromm E., Wade L. (1970). Melanin, adrenaline and the legacy of fear. J. Hered. 61: 81-88.

Keeler, C. (1975). Genetics of behaviour variations in color phases of the red fox. In: M.W.Fox (Editor). The Wild Canids. Van Nostrand Reinhold, New York. p. 399-417.

Lowe, A., Harris S., Ashton P. (2004). Ecological Genetics: Design, analysis, and application. Oxford, UK, Blackwell Publishing Ltd.
Nei, M. (1972). Genetic distance between populations. Amer. Natur. 106: 283-29

Peña-Cruz, A.F., Sandoval, S., Patiño, A., Bedoya, M., Rodríguez, A., Orjuela, J., Ortega, A., López, J., Molina, E., Guzmán, A., Gil, J., Cárdenas, H. (2015). Genetic Analysis of the Cat Population of North and South of Cali, Colombia. Acta Biol. Col. 20 (1): 109-116.

Ruiz-García, M. \& D. Álvarez. (1999). Análisis filogenético de 21 poblaciones latinoamericanas de gatos mediante 10 loci morfológicos utilizando métodos de matrices de distancias genéticas y de máxima parsimonia. Bol. Real Soc. Españ. Hist. Nat. 95: 143-168.

Ruiz M. \& Álvarez D. (2003). Posible origen europeo de seis poblaciones latinoamericanas de gatos y no existencia de paralelismo con el modelo colonizador británico al utilizar genes del pelaje y microsatélites. Act. Zool. Mex. 89: $261-286$

Ruiz-García M., Álvarez D., Shostell J. M. (2005). Population genetic analysis of cat populations from Mexico, Colombia, Bolivia, and the Dominican Republic: Identification of different gene pools in Latin America. J. Genet. 84: 147-171.

Ruiz-García, M. 2010a. Micro-geographical genetic structure of Inia geoffrensis in the Napo-Curaray River basin by means of Chesser's models. In M. Ruiz-García, J. Shostell (editors). Biology, Evolution, and Conservation of River Dolphins within South America and Asia. Nova Science, Nueva York, EEUU. p. 131-160.

Slatkin, M. (1987). Gene flow and the geographic structure of natural populations. Science. 236: 787e792.

Tamura, K., Peterson, D., Peterson, N., Stecher, G., Nei, M., Kumar, S. (2011). MEGA 5: Molecular evolutionary genetics using maximum likelihood, evolutionary distance, and maximum parsimony methods. Mol. Biol. Evol. 28 (10): 2731-2739.

Todd, N.B. (1977a). Cats and Commerce. Scien. Amer. 237: 100-107.

Yeh, F.C., Boyle, T., Ye, Z., Xiyan, J.M. (1999). POPGENE Version1.31: Microsoft Windows-based freeware for population genetic analysis. University of Alberta and Center for International Forestry Research. 